\title{
Bubble rise dynamics in a viscoplastic material
}

\author{
Manoj Kumar Tripathi, Kirti Chandra Sahu, George Karapetsas ${ }^{\dagger}$ and \\ Omar K. Matar ${ }^{\dagger \dagger}$ \\ Department of Chemical Engineering, Indian Institute of Technology Hyderabad, \\ Yeddumailaram 502 205, Andhra Pradesh, India, \\ ${ }^{\dagger}$ Department of Mechanical Engineering, University of Thessaly, Volos 38334, Greece, \\ ${ }^{\dagger}$ Department of Chemical Engineering, Imperial College London, South Kensington \\ Campus, London, SW7 2AZ, UK
}

\section{Abstract}

The axisymmetric dynamics of a bubble rising in a Bingham fluid under the action of buoyancy is studied. The Volume-of-Fluid (VOF) method is used to solve the equations of mass and momentum conservation, coupled to an equation for the volume fraction of the Bingham fluid. A regularised constitutive model is used for the description of the viscoplastic behaviour of the material. The numerical results demonstrate that the rise dynamics are complex for large yield stresses, and for weak surface tension. Under these conditions, for which the bubble is highly deformable, the rise is unsteady and is punctuated by periods of rapid acceleration which separate stages of quasi-steady motion. During the acceleration periods, the bubble aspect ratio exhibits oscillations about unity, whose amplitude and wavelength increase with increasing yield stress and decreasing surface tension. These oscillations are accompanied by the alternating formation and destruction of unyielded

\footnotetext{
1o.matar@imperial.ac.uk 
zones at the bubble equator, as the bubble appears to "swim" upwards. Keywords: Multiphase flow, Non-Newtonian, Immiscible fluids, Bubbles, Numerical simulation.

\section{Introduction}

The motion of droplets in fluids that exhibit yield stress is important in many engineering applications, including food processing, oil extraction, waste processing and biochemical reactors. Yield stress fluids or viscoplastic materials flow like liquids when subjected to stress beyond some critical value, the so-called yield stress, but behave as a solid below this critical level of stress; detailed review on yield stress fluids can be found in [1, 2]. As a result the gravity-driven bubble rise in a viscoplastic material is not always possible as in the case of Newtonian fluids but occurs only if buoyancy is sufficient to overcome the material's yield stress $[3,4]$; the situation is also similar for the case of a settling drop or solid particle [5].

The first constitutive law proposed to describe this material behavior is the Bingham model [6] which was later extended by Herschel \& Bulkley [7] to take into account the effects of shear-thinning (or thickening). According to this model the material can be in two possible states; it can be either yielded or unyielded, depending on the level of stress it experiences. As the common boundary of the two distinct regions the so-called yield surface is approached, the exact Bingham model becomes singular. In simple flows this singularity does not generate a problem, but, in more complex flows the dis- 
continuous behaviour of the Bingham model may pose significant difficulties due to the fact that in most cases the yield surface is not known a priori but must be determined as part of the solution. Nevertheless, there are examples of successful analysis of two-dimensional flows using this model at the expense of relatively complicated numerical algorithms [5, 8-10]. A simpler way to overcome these difficulties is to modify the Bingham constitutive equation in order to produce a non-singular constitutive law, by introducing a 'regularization' parameter [11]. This method has been used with success by several researchers in the past $[4,12-15]$ and when used with caution can give significant insight in the behaviour of viscoplastic materials.

The motion of air bubbles in viscoplastic materials has attracted the interest of many research groups in the past. The first reported experimental study on rising bubbles in a viscoplastic material was done by Astarita \& Apuzzo [16] who reported bubble shapes and velocities in Carbopol solutions. They observed that curves of bubble velocity vs bubble volume for viscoplastic liquids had an abrupt change in slope at a critical value of bubble volume that depended on the concentration of Carbopol in the solution, i.e. the yield stress of the material. Many years later, Terasaka \& Tsuge [17] used xanthan gum and Carbopol solutions to examine the formation of bubbles at a nozzle and derived an approximate model for bubble growth. Dubash \& Frigaard [18] also performed experiments with Carbopol solutions and were able to comfirm the observations of Astarita \& Apuzzo [16] on the existence of a critical bubble radius required to set it in motion and noted 
that the entrapment conditions are affected significantly by surface tension. It is also noteworthy that the observed bubble shapes inside a vertical pipe were different from [16] exhibiting a cusped tail, resembling much the inverted teardrop shapes often found inside a viscoelastic medium [16, 19, 20]. Similar bubble shapes have been found in the experimental studies by Sikorski et al. [21] and Mougin et al. [22], using Carbopol solutions of different concentrations. The latter authors also studied the significant role of internal trapped stresses within a Carbopol gel on the trajectory and shape of the bubbles; their findings were in agreement with an earlier study presented by Piau [23].

From a theoretical point of view, Bhavaraju et al. [24] performed a perturbation analysis in the limit of small yield stress for a spherical air bubble. Stein \& Buggish [25] were interested on the mobilization of bubbles by setting an oscillating external pressure and provided analytical solutions along with some experimental data; the latter suggested that larger bubbles tend to rise faster than smaller bubbles at similar amplitudes. Dubash \& Frigaard [3] employed a variational method to estimate the conditions under which bubbles should remain static. These estimations, however, were characterized as conservative, in the sense that they provide a sufficient but not necessary condition. A detailed numerical study of the steady bubble rise, using the regularized Papanastasiou model [26], has been performed by Tsamopoulos et al. [4]. These authors presented mappings of bubble and yield surface shapes for a wide range of dimensionless parameters, taking into account the 
effects of inertia, surface tension and gravity. Moreover, they were able to evaluate the conditions for bubble entrapment. Their work was followed by the study of Dimakopoulos et al. [10] who used the augmented Lagrangian method to obtain a more accurate estimation of the stopping conditions. It was shown that the critical Bingham number, $B n$, does not depend on the Archimedes number in accordance with Tsamopoulos et al. [4], but depends non-monotonically on surface tension. We should note that in both studies the shape of the bubble near critical conditions could not reproduce the inverted teardrop shapes seen in experiments [18, 21, 22] and raised questions whether this is due to elasticity, thixotropy or wall effects. Besides the steady solutions it is also interesting to investigate the bubble dynamics through time-dependent simulations. This was done by Potapov et al. [27] and Singh \& Denn [28] using the VOF method and the level-set method, respectively. Singh \& Denn [28] considered creeping flow conditions and performed simulations for single and multiple bubbles. It was shown that multiple bubbles and droplets can move inside the viscoplastic material under conditions that a single bubble or droplet with similar properties would have been trapped unable to overcome the yield stress. Potapov et al. [27] also studied the case of a single or two interacting bubbles but also took into account the effect of inertia, albeit for a low Reynolds number. For the parameter range that they have used the single bubble always reached a quasi-steady state. We should note at this point that for some cases (e.g. for high values of the Archimedes number) Tsamopoulos et al. [4] were not able to calculate steady shapes 
which is probably an indication that the flow may become time-dependent. Even in the cases where a steady solution could be obtained it is not certain that this solution is stable. Therefore a question that arises is whether for some parameter values it is possible to get a time-dependent solution and what would be the dynamics of the bubble flow in this case. This is the question that our paper will attempt to address.

In this paper, we study the buoyancy-driven rise of a bubble inside an infinite viscoplastic medium, assuming axial symmetry. To account for the viscoplacity we consider the regularized Herschel-Bulkley model. We employ the Volume-of-Fluid method to follow the deforming bubble along the domain. Our results indicate that in the presence of inertia and in the case of weak surface tension the bubble does not reach a steady state and the dynamics may become complex for sufficiently high yield stress of the material.

The rest of the paper is organized as follows. In Section 2, we outline the governing equations, and in Section 3 discuss our numerical results. Finally, concluding remarks are given in Section 4.

\section{Formulation}

\subsection{Governing equations}

We consider the rise of a bubble (Newtonian fluid 'B') in a viscoplastic material (fluid 'A') under the action of buoyancy within a cylindrical domain of diameter $H$ and height $L$, as shown in Fig. 1. We use an axisymmetric, cylindrical coordinate system, $(r, z)$, to model the flow dynamics, in which $r$ and $z$ denote the radial and axial coordinates, respectively, the latter being 
aligned in the opposite direction to gravity. The bubble is initially present at a distance $z_{i}$ above the bottom of the domain located at $z=0$. The governing equations of the problem correspond to those of mass and momentum conservation:

$$
\begin{gathered}
\nabla \cdot \mathbf{u}=0 \\
\rho\left[\frac{\partial \mathbf{u}}{\partial t}+\mathbf{u} \cdot \nabla \mathbf{u}\right]=-\nabla p+\nabla \cdot\left[\mu\left(\nabla \mathbf{u}+\nabla \mathbf{u}^{T}\right)\right]+\mathbf{F},
\end{gathered}
$$

and the following equation for the volume fraction, $c$, of the fluid $A$.

$$
\frac{\partial c}{\partial t}+\mathbf{u} \cdot \nabla c=0
$$

In the above equations, $\mathbf{u}=\left(u_{r}, u_{z}\right)$ denotes the velocity field in which $u_{r}$ and $u_{z}$ represent the radial and axial velocity components, respectively, $p$ is the pressure field, $t$ denotes time, and $\mathbf{F}$ is the combined body and surface forces per unit volume, which include the gravity and surface tension forces given by:

$$
\mathbf{F}=\delta \sigma \kappa \mathbf{n}-\rho g \mathbf{j}
$$

here, $j$ denotes the unit vector along the vertical direction, $\sigma$ and $g$ represent the (constant) interfacial tension and gravitational acceleration, respectively, $\delta$ is the Dirac delta function, and $\kappa=\nabla \cdot \mathbf{n}$ is the interfacial curvature in which $\mathbf{n}$ is the outward-pointing unit normal to the interface.

The density, $\rho$, and the viscosity, $\mu$, are assumed to depend on the volume fractions of the fluids:

$$
\rho=c \rho_{A}+(1-c) \rho_{B}
$$




$$
\mu=c \mu_{A}+(1-c) \mu_{B}
$$

where $\rho_{i}$ and $\mu_{i}(i=A, B)$ denote the densities and the dynamic viscosities, respectively, of the surrounding Bingham fluid ('A') and the Newtonian fluid ('B') inside the bubble. Here, $\rho_{i}(i=A, B)$ and $\mu_{B}$ are considered to be constant whereas $\mu_{A}$ is given by

$$
\mu_{A}=\frac{\tau_{0}}{\Pi+\epsilon}+\mu_{0}(\Pi+\epsilon)^{n-1}
$$

where $\tau_{0}$ and $n$ are the yield stress and flow index, respectively, $\epsilon$ is a small regularization parameter, and $\mu_{0}$ is the fluid consistency; $\Pi=\left(E_{i j} E_{i j}\right)^{1 / 2}$ is the second invariant of the strain rate tensor, wherein $E_{i j} \equiv \frac{1}{2}\left(\partial u_{i} / \partial x_{j}+\partial u_{j} / \partial x_{i}\right)$. In the simulations to be presented in this paper and after careful evaluation, we have chosen the value of $\epsilon$ down to $10^{-4}$ in order to neither affect the yield surface by overly increasing $\epsilon$ nor produce numerical instabilities or stiff equations by decreasing it further; similar values for $\epsilon$ have been used earlier by Singh and Denn [28]. Finally, we set $n=1$ henceforth so that our non-Newtonian fluid corresponds to a Bingham plastic and the effect of a shear-dependent viscosity will be ignored for the purposes of the present study. An important ingredient of every study that concerns the flow of a viscoplastic material is the determination of the position of the yield surface and when using a regularized model this can be achieved a posteriori by using the following criterion:

$$
\text { yielded material: } T>\tau_{0} \text {, }
$$


unyielded material: $T \leq \tau_{0}$,

where $T$ denotes the second invariant of the stress tensor in material 'A',

$$
T=\left[\frac{1}{2} \tau_{i j} \tau_{j i}\right]^{1 / 2}
$$

and $\tau_{i j}$ is given by

$$
\tau_{i j}=\mu_{A} E_{i j}
$$

\subsection{Scaling}

The following scaling is employed in order to render the governing equations dimensionless:

$$
\begin{gathered}
(r, z)=R(\widetilde{r}, \widetilde{z}), t=\frac{R}{V} \widetilde{t}, \mathbf{u}=V \tilde{\mathbf{u}}, p=\rho_{A} V^{2} \widetilde{p}, \\
\mu=\mu_{0} \widetilde{\mu}, \rho=\rho_{A} \widetilde{\rho},
\end{gathered}
$$

where $R$ is the initial radius of the bubble, the velocity scale is $V=\sqrt{g R}$, and the tildes designate dimensionless quantities. After dropping tildes from all nondimensional variables, the governing dimensionless equations are given by

$$
\begin{gathered}
\nabla \cdot \mathbf{u}=0, \\
\frac{\partial \mathbf{u}}{\partial t}+\mathbf{u} \cdot \nabla \mathbf{u}=-\nabla p+\frac{1}{R e} \nabla \cdot\left[\mu\left(\nabla \mathbf{u}+\nabla \mathbf{u}^{T}\right)\right]+\mathbf{F}, \\
\frac{\partial c}{\partial t}+\mathbf{u} \cdot \nabla c=0,
\end{gathered}
$$


where $R e \equiv \rho_{A} V R / \mu_{0}$ denotes the Reynolds number. The dimensionless density is given by

$$
\rho=c+(1-c) \rho_{r}
$$

where $\rho_{r} \equiv \rho_{B} / \rho_{A}$. The dimensionless viscosity, $\mu$, is given by:

$$
\mu=\left(\frac{B n}{\Pi+\epsilon}+m(\Pi+\epsilon)^{n-1}\right) c+(1-c) \mu_{r},
$$

where $B n \equiv \tau_{0} R / \mu_{0} V$ is the Bingham number; $m=(V / R)^{n-1}$ and $\mu_{r}=$ $\mu_{B} / \mu_{0}$. The position of the yield surface is determined by evaluating the dimensionless second stress tensor, $T$, inside fluid 'A' and finding the locus of points for which $T=B n$.

Finally, in Eq. 14, the combined body and surface forces, F, is given by

$$
\mathbf{F}=\delta \frac{\nabla \cdot \mathbf{n}}{B o} \mathbf{n}-\rho \mathbf{j}
$$

where $B o\left(\equiv \rho_{A} g R^{2} / \sigma_{0}\right)$ is the Bond number. A discussion of the results is presented next.

\section{Results}

\subsection{Numerical method and validation}

We use a finite-volume flow solver [29] that solves Eqs. (13)-(15) on a collocated grid. It is to be noted that the density and the viscosity ratios in the problem considered in the present study, $\rho_{r}$ and $\mu_{r}$, are very large, which can create spurious currents at the interface. The open-source finite-volume fluid flow solver [30] (Gerris) minimizes this problem by using 
the balanced-force continuum surface force formulation for the calculation of surface tension. This solver is also equipped with adaptive mesh refinement capabilities. The results obtained using this solver are cross-checked using a bespoke finite-volume staggered grid code based on diffuse-interface technique. The pressure and the volume fraction of the outer fluid are defined at the cell-centres, and the velocity components are defined at the cell faces, respectively. In our code weighted-essentially-non-oscillatory (WENO), and central difference schemes are used to discretize the advective and diffusive terms in Eq. (15), respectively. In order to achieve second-order accuracy, the Adams-Bashforth and the Crank-Nicholson methods are used to discretize the advective and dissipation terms in Eq. (14), respectively.

We assume that the flow is symmetrical about the axis $r=0$. Neumann boundary conditions are imposed at the rest of the boundaries. In Fig. 2, we present an illustration of the convergence of the numerical solutions upon mesh refinement. The parameters chosen for this case are $R e=70.71$, $B n=14.213, \mu_{r}=0.01, \rho_{r}=0.001, m=1$ and $B o=30$. The reader is also

referred to the supplementary material of Tripathi et al. [29] for an extensive validation of the present code.

\subsection{Discussion}

We begin the discussion of our results by examining the dependence of the results on the regularisation parameter, $\epsilon$, used in the viscosity model for fluid 'A', given by Eq. (17), for $R e=70.71, B n=14.213, \mu_{r}=0.01$, $\rho_{r}=0.001, m=1$ and $B o=30$. In Fig. 3, we show that the bubble rise 
is accompanied by its deformation and the development of a yielded region which surrounds the bubble at $t=10$, in which the stress generated from the bubble motion is sufficient to exceed the yield stress in fluid 'A'; this region is itself surrounded by unyielded fluid. Also shown in Fig. 3 is the formation of three small unyielded regions: two at the bubble equator, and one near the dimple located at the bubble base; similar predictions have been presented by Tsamopoulos et al. [4] using the Papanastasiou model [26].

It is seen that the dependence of the shapes of the bubble and the yielded region surrounding it, as well as the extent of the unyielded regions immediately adjacent to the bubble becomes progressively weaker with increasing $\epsilon$. At this point, it should be noted that decreasing the value of $\epsilon$ the system of equations becomes stiffer and more difficult to handle numerically. This may also result in the appearance of numerical noise and therefore very small values of $\epsilon$ should actually be avoided. A more accurate evaluation of the yield surface position is possible, as was shown recently by Dimakopoulos et al. [10] using the augmented Langrangian method at the expense of a significantly more complex numerical algorithm. Nevertheless, for the purposes of this study, the calculated yield surfaces are considered to be reasonably accurate. We have also found that the time evolution of the bubble aspect ratio and its centre of gravity, $z_{C G}$, exhibit a similar dependence on $\epsilon$ and become virtually indistinguishable with decreasing $\epsilon$, as shown in Fig. 4. Thus, the rest of the results discussed in this paper have been generated using $\epsilon=0.001$. 
Next, we study the bubble rise dynamics by examining the temporal evolution of the bubble aspect ratio and centre of gravity for varying Bingham number, $B n$, with $R e=7.07, B o=10, \mu_{r}=0.01, \rho_{r}=0.001$, and $m=1$. It is seen in Fig. 5 that for low $B n$ values, which reflect the presence of a weak yield stress, the bubble undergoes severe deformation at relatively early times before assuming a constant aspect ratio. More specifically, for $B n=0.071$ the aspect ratio is found to be approximately equal to 0.48 in good agreement with the predictions given by Tsamopoulos et al. [4]. We also found that, as expected, the rise velocity of the bubble decreases with $B n$ due to the increased resistance associated with the larger yield stresses (see Fig. 5).

In the low $B n$ range, the bubble achieves a constant rise speed rapidly, as shown by the linear dependence of $z_{C G}$ on time. In particular for $B n=0.071$ the calculated terminal velocity is approximately equal to 0.765 in agreement with the predicted value of 0.75 given in Tsamopoulos et al. [4]. The extent of bubble deformation and rise speed decrease with increasing $B n$ for $B n$ less than unity for the parameters used to generate the results shown in Fig. 5; the same trend was also found in Tsamopoulos et al.[4]. For higher $B n$ values, e.g. $B n=0.99$, we notice that the bubble aspect ratio (1.05) and terminal velocity (0.226) differ significantly from the predictions of Tsamopoulos et al. [4], i.e. 1.25 and 0.07 , respectively. The difference cannot be attributed to the finite viscosity of the fluid since, as shown in Fig. 6, increasing the viscosity ratio, $\mu_{r}$, leads to the decrease of the rise velocity. We notice though that 
even at late times the deformation of the bubble has not reached a steady state (see Fig. 6b) and continues to change. As shown in Fig. 5b, the latter effect is more prominent for even higher values of the $B n$ number where we see clearly that the flow does not reach a steady state and that the bubble aspect ratio exhibits finite amplitude oscillations. These oscillations in the bubble deformation may lead to yielding of the surrounding material and thereby could be responsible for the enhancement of the bubble motion.

Fig. 7 depicts the spatio-temporal evolution of the shape of the bubble and its surrounding unyielded region as a function of $B n$ for the same parameters used to generate Fig. 5. Inspection of this figure shows that the extent of the unyielded region increases with $B n$, as expected, and for $B n<1$, the bubble widens as it rises, which is consistent with the results shown in Fig. 5(b) for the same range of parameter values and in accordance with the findings of Tsamopoulos et al. [4]. These shapes become steady with increasing time. For $B n=1.34$, it is evident that the bubble aspect ratio exceeds unity, which is also consistent with Fig. 5(b), likely brought about by the confinement due to the smaller yielded region associated with this value of $B n$; it is also evident that the shapes of the bubble and unyielded regions do not achieve a steady-state in this case.

In Fig. 8 we show contour plots of the radial and axial components of the velocity field for $B n=0,0.35,0.99,1.34$, and the rest of the parameters remain unchanged from those of Fig. 5. The case with $B n=0$ corresponds to the Newtonian case. It is clearly seen that the radial and axial velocity 
components exhibit stagnation contours that separate regions of outward and inward, and upwards and downwards motion, respectively; at regions where the radial motion of the fluid is negligible, unyielded zones are likely to occur. The stagnation contour associated with the vertical component moves progressively closer to the interface with increasing $B n$; for the largest $B n$ studied, it is evident that the regions nearest the top and bottom of the bubble move upwards, while the remaining regions move downwards leading to bubble elongation. The stagnation contour associated with the radial component emanates from rightmost bubble edge at a negative angle to the horizontal in the Newtonian case. This contour becomes essentially horizontal and the bubble, whose bottom is dimpled in the Newtonian case, becomes well-rounded with inceasing $B n$ as the bubble becomes flatter at the equatorial plane.

It is also important to study the effect of bubble deformability on its dynamics; this is done by varying the Bond number, $B o$, which reflects the relative significance of surface tension to gravtiational forces. In Fig. 9, it is seen clearly that for low Bo, for which surface tension forces are dominant, bubble deformation is small and its rise speed is constant, increasing with $B o$. For larger $B o$, however, the bubble dynamics gain in complexity. The bubble appears to undergo sudden acceleration between periods of constant rise speed; these periods become shorter and the magnitude of the acceleration increases with $B n$, as shown in Fig. 9a. This $z_{C G}$ dynamics is associated with large bubble deformation as can be ascertained upon inspection of Fig. 9b: 
the aspect ratio undergoes nonlinear oscillations about unity as the bubble 'swims' upwards, whose wavelength and amplitude increase with $B o$.

In order to rationalise the results presented in Fig. 9 and further highlight the role of bubble deformation in the 'swimming' motion discussed, we show in Fig. 10 the spatio-temporal evolution of the shape of the bubble and the unyielded regions for $B o=1$ and $B o=30$ while the rest of the parameters remain unchanged from those of Fig. 9. It is seen that for $B o=30$, at relatively early times unyielded regions are situated in the equatorial region of the bubble, and the bubble aspect ratio is close to unity. With increasing time, the extent of the unyielded regions decreases due to the shear stress associated with the bubble acceleration and the bubble elongates as it rises through a yielded region of increasing size. The bubble then decelerates to a constant rise speed, its aspect ratio decreases, and the decrease in shear stress in the vicinity of the interface leads to the development of unyielded zones in the equatorial and south pole regions; the former become more pronounced with increasing time, and the bubble aspect ratio decreases below unity as the bubble decelerates. The process is then repeated. In contrast, no such process is evident in the case of $B o=1$ for which the bubble appears to suffer negligible deformation and the size of the unyielded regions remains largely unaltered.

In Fig. 11, we show the effect of $B o$ on the contour plots of the radial and axial velocity components for $B o=1$ and $B o=30$; these plots are shown for $t=6$ and $t=8.5$ that correspond to the times at which the bubble achieves 
its maximal and minimal aspect ratio for $B o=30$, respectively. As can be seen from this figure, the magnitude of both components remains essentially unchanged for the $B o=1$ case, while, for the same times in the $B o=30$ case, the axial and radial velocity components dominate at $t=6$ and $t=8.5$, resulting in bubble elongation, and flattening and dimpling, respectively.

\section{Conclusions}

In this paper, we have examined the axisymmetric dynamics of bubble rise in Bingham fluids. We have used an open-source finite-volume flow solver, Gerris based on volume-of-fluid methodology to study the flow, which involves the numerical solution of the equations of mass and momentum conservation, and an equation of the volume fraction of the Bingham fluid. The momentum equation accounts for surface tension and gravitational effects, while the density and viscosity are volume fraction-weighted with respect to the corresponding quantities of the two fluids. For the Bingham fluid, the

formula for the viscosity contains a regularisation parameter; convergence of our results was achieved upon mesh-refinement and reduction of the magnitude of this parameter to sufficiently small values.

Our numerical results indicate that in the presence of weak yield stress the bubble achieves a constant rise speed relatively rapidly, whilst its aspect ratio, defined as the ratio of its height to its width asymptotes to a value less than unity; unyielded zones are confined to regions that surround but are not immediately adjacent to the bubble. With increasing yield stress, the bubble 
rise is unsteady, and the bubble aspect ratio exhibits oscillations above a value that exceeds unity. Unyielded zones near the equatorial and south pole regions of the bubble have also been observed to form for sufficiently large yield stress in agreement with earlier studies in the literature $[4,10]$.

We have also shown that bubble deformation has a profound impact on the dynamics. In the case of strong surface tension, the rise is steady and the bubble suffers negligible deformation. For weak surface tension, however, the rise is unsteady, periods of approximately constant rise speed are separated by rapid acceleration stages that coincide with oscillations in the bubble aspect ratio about unity whose amplitude increases with decreasing surface tension. These oscillations also coincide with the formation and destruction of unyielded zones in the equatorial regions. The motion executed by the bubble for this range of parameters resembles 'swimming' as the bubble appears to grab hold of the unyielded zones to propel itself upwards.

\section{Acknowledgements}

KS thanks the Indian National Science Academy for their financial support.

[1] R. B. Bird, G. C. Dai, B. J. Yarusso, The rheology and flow of viscoplastic materials, Rev. Chem. Eng. 1 (1982) 1-70.

[2] H. A. Barnes, The yield stressa review or everything flows?, Journal of Non-Newtonian Fluid Mechanics 81 (1999) 133-178.

[3] N. Dubash, I. Frigaard, Conditions for static bubbles in viscoplastic fluids, Physics of Fluids (2004) 4319-4330. 
[4] J. Tsamopoulos, Y. Dimakopoulos, N. Chatzidai, G. Karapetsas, M. Pavlidis, Steady bubble rise and deformation in newtonian and viscoplastic fluids and conditions for bubble entrapment, Journal of Fluid Mechanics 601 (2008) 123-164.

[5] A. N. Beris, J. A. Tsamopoulos, R. C. Armstrong, R. A. Brown, Creeping motion of a sphere through a bingham plastic, Journal of Fluid Mechanics 158 (1985) 219-244.

[6] E. C. Bingham, Fluidicity and Plasticity, McGraw-Hill, New York, 1922.

[7] W. H. Herschel, P. Bulkley, Konsistenzmessungen von gummibenzollsungen, Kolloid Zeitschrift 39 (1926) 291-300.

[8] N. Roquet, P. Saramito, An adaptive finite element method for bingham fluid flows around a cylinder, Computer Methods in Applied Mechanics and Engineering 192 (2003) 3317-3341.

[9] M. A. Moyers-Gonzalez, I. A. Frigaard, Numerical solution of duct flows of multiple visco-plastic fluids, Journal of Non-Newtonian Fluid Mechanics 122 (2004) 227-241.

[10] Y. Dimakopoulos, M. Pavlidis, J. Tsamopoulos, Steady bubble rise in HerschelBulkley fluids and comparison of predictions via the augmented lagrangian method with those via the papanastasiou model, Journal of Non-Newtonian Fluid Mechanics 200 (2013) 34-51. 
[11] I. Frigaard, C. Nouar, On the usage of viscosity regularisation methods for visco-plastic fluid flow computation, Journal of Non-Newtonian Fluid Mechanics 127 (2005) 1-26.

[12] G. R. Burgos, A. N. Alexandrou, V. Entov, On the determination of yield surfaces in Herschel-Bulkley fluids, Journal of Rheology 43 (1999) 463.

[13] J. Blackery, E. Mitsoulis, Creeping motion of a sphere in tubes filled with a bingham plastic material, Journal of Non-Newtonian Fluid Mechanics 70 (1997) 5977.

[14] M. Beaulne, E. Mitsoulis, Creeping motion of a sphere in tubes filled with herschel-bulkley fluids, Journal of Non-Newtonian Fluid Mechanics 72 (1997) 5571.

[15] J. Papaioannou, G. Karapetsas, Y. Dimakopoulos, J. Tsamopoulos, Injection of a viscoplastic material inside a tube or between two parallel disks: Conditions for wall detachment of the advancing front, Journal of Rheology 53 (2009) 1155-1191.

[16] G. Astarita, G. Apuzzo, Motion of gas bubbles in non-newtonian liquids, AIChE Journal 11 (5) (1965) 815820.

[17] K. Terasaka, H. Tsuge, Bubble formation at a nozzle submerged in viscous liquids having yield stress, Chemical Engineering Science 56 (2001) $3237-3245$. 
[18] N. Dubash, I. Frigaard, Propagation and stopping of air bubbles in carbopol solutions, Journal of Non-Newtonian Fluid Mechanics 142 (2007) $123-134$.

[19] C. Mlaga, J. Rallison, A rising bubble in a polymer solution, Journal of Non-Newtonian Fluid Mechanics 141 (2007) 59-78.

[20] C. Pilz, G. Brenn, On the critical bubble volume at the rise velocity jump discontinuity in viscoelastic liquids, Journal of Non-Newtonian Fluid Mechanics 145 (2007) 124-138.

[21] D. Sikorski, H. Tabuteau, J. R. de Bruyn, Motion and shape of bubbles rising through a yield-stress fluid, Journal of Non-Newtonian Fluid Mechanics 159 (2009) 10-16.

[22] N. Mougin, A. Magnin, J.-M. Piau, The significant influence of internal stresses on the dynamics of bubbles in a yield stress fluid, Journal of Non-Newtonian Fluid Mechanics 171-172 (2012) 42-55.

[23] J. Piau, Carbopol gels: Elastoviscoplastic and slippery glasses made of individual swollen sponges, Journal of Non-Newtonian Fluid Mechanics 144 (2007) 1-29.

[24] S. M. Bhavaraju, R. A. Mashelkar, H. W. Blanch, Bubble motion and mass transfer in non-newtonian fluids: Part i. single bubble in power law and bingham fluids, AIChE Journal 24 (1978) 1063-1070. 
[25] S. Stein, H. Buggisch, Rise of pulsating bubbles in fluids with a yield stress, ZAMM - Journal of Applied Mathematics and Mechanics / Zeitschrift fr Angewandte Mathematik und Mechanik 80 (2000) 827834.

[26] T. C. Papanastasiou, Flows of materials with yield, J. Rheol. 31 (1987) $385-404$.

[27] A. Potapov, R. Spivak, O. M. Lavrenteva, A. Nir, Motion and deformation of drops in bingham fluid, Industrial \& Engineering Chemistry Research 45 (21) (2006) 6985-6995.

[28] J. P. Singh, M. M. Denn, Interacting two-dimensional bubbles and droplets in a yield-stress fluid, Physics of Fluids 20 (2008) 040901.

[29] M. K. Tripathi, K. C. Sahu, R. Govindarajan, Why a falling drop does not in general behave like a rising bubble, Nature Scientific Reports 4 (2014) 4771.

[30] S. Popinet, Gerris: a tree-based adaptive solver for the incompressible euler equations in complex geometries, Journal of Computational Physics 190 (2003) 572-600. 


\section{List of figures}

Fig. 1: Schematic diagram of a bubble of fluid 'B' rising inside a Bingham fluid 'A' under the action of buoyancy. The bubble is placed at $z=z_{i}$; the value of $H, L$ and $z_{i}$ are taken to be $20 R, 48 R$, and $10.5 R$, respectively. Initially the aspect ratio of the bubble, $h / w$ is 1 , wherein $h$ and $w$ are the height and width of the bubble.

Fig. 2: The shape of the bubble along with the mesh are shown for (a) finer and (b) coarser grids. Adaptive grid refinement has been used in the interfacial and yielded regions. The smallest mesh size in the finer and coarser grids are 0.015 and 0.0625 , respectively. Note that the finer grid has been used to generate the results presented in the subsequent figures. The parameter values are $R e=70.71, B n=14.213, \mu_{r}=0.01, \rho_{r}=0.001, m=1$ and $B o=30$.

Fig. 3: The unyielded region in the non-Newtonian fluid (shown in black) for different values of the regularized parameter, $\epsilon$ : (a) $\epsilon=0.01$, (b) $\epsilon=0.001$, (c) $\epsilon=0.0001$. The rest of the parameter values are $R e=70.71, B n=$ 14.213, $\mu_{r}=0.01, \rho_{r}=0.001, m=1$ and $B o=30$.

Fig. 4: (a) Temporal variation of the center of gravity, (b) the aspect ratio of the bubble for different values of $\epsilon$. The rest of the parameter values are the same as those used to generate Fig. 3. 
Fig. 5: (a) Temporal variation of the center of gravity, (b) the aspect ratio of the bubble for different values of $B n$. The parameter values are $R e=7.071$, $\mu_{r}=0.01, \rho_{r}=0.001, m=1$ and $B o=10$.

Fig. 6: (a) Temporal variation of the center of gravity, (b) the aspect ratio of the bubble for different values of $\mu_{r}$. The parameter values are $R e=7.071$, $B n=0.99, \rho_{r}=0.001, m=1$ and $B o=10$.

Fig. 7: The evolution of the shape of the bubble (shown by red lines) and the unyielded region in the non-Newtonian fluid (shown in black) for different values of Bingham number. The results of the Newtonian case are shown for the comparison purpose. The rest of the parameter values are the same as those used to generate Fig. 5 .

Fig. 8: Contour plots for the radial (right) and axial (left) velocity components for (a) $B n=0$ at $t=6$ (Newtonian case), (b) $B n=0.354$ at $t=6$, (c) $B n=0.99$ at $t=20$ and (d) $B n=1.34$ at $t=20$. In each panel the shape of the bubble is shown by red line. The rest of the parameter values are the same as those used to generate Fig. 5.

Fig. 9: (a) Temporal variation of the center of gravity, (b) the aspect ratio of the bubble for different values of $B o$. The rest of the parameter values are 
$R e=70.71, B n=14.213, \mu_{r}=0.01, \rho_{r}=0.001$, and $m=1$.

Fig. 10: The evolution of the shape of the bubble (shown by red lines) and the unyielded regions in the Bingham fluid (shown in black) for different values of $B$ o. The rest of the parameter values are the same as those used to generate Fig. 9.

Fig. 11: Contour plots for the radial (right) and axial (left) velocity components for (a) $B o=1$ at $t=6$, (b) $B o=1$ at $t=8.5$, (c) $B o=30$ at $t=6$ and (d) $B o=30$ at $t=8.5$. In each panel the shape of the bubble is shown by red line. The rest of the parameter values are the same as those used to generate Fig. 9. 


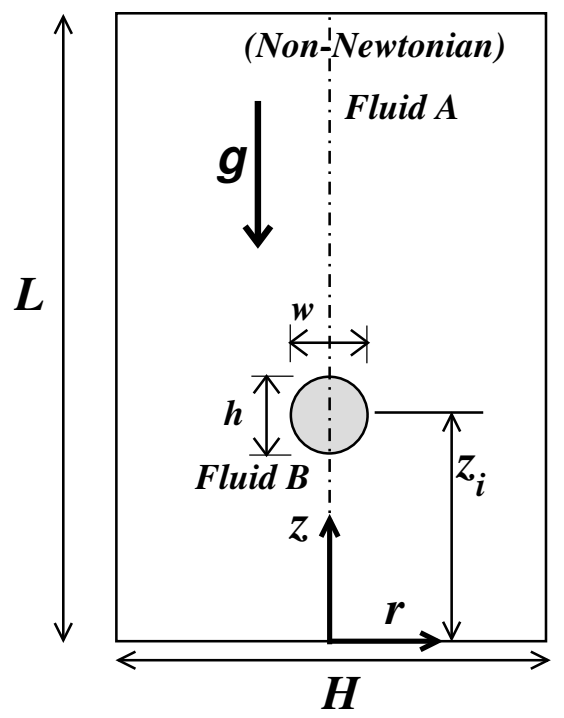

Figure 1: Schematic diagram of a bubble of fluid 'B' rising inside a Bingham fluid 'A' under the action of buoyancy. The bubble is placed at $z=z_{i}$; the value of $H, L$ and $z_{i}$ are taken to be $20 R, 48 R$, and $10.5 R$, respectively. Initially the aspect ratio of the bubble, $h / w$ is 1 , wherein $h$ and $w$ are the height and width of the bubble. 
(a)

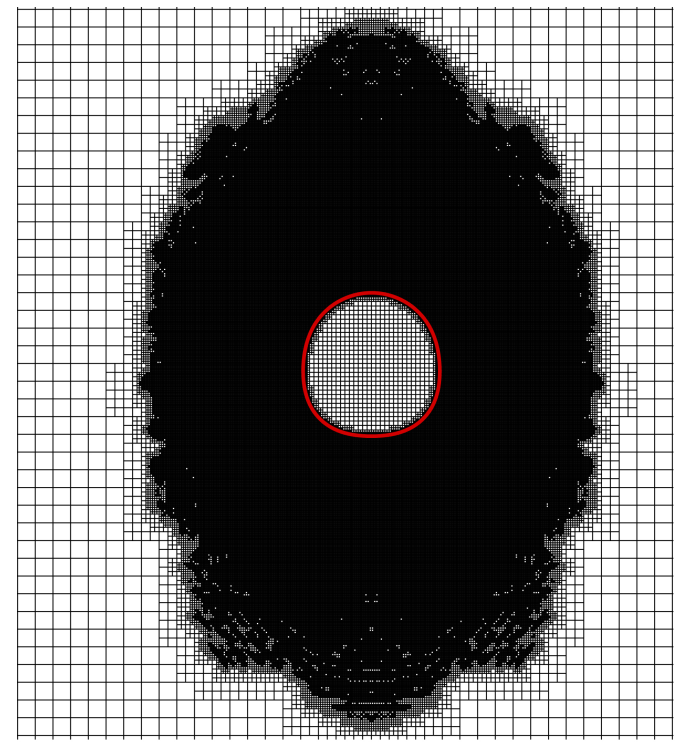

Finer grid (b)

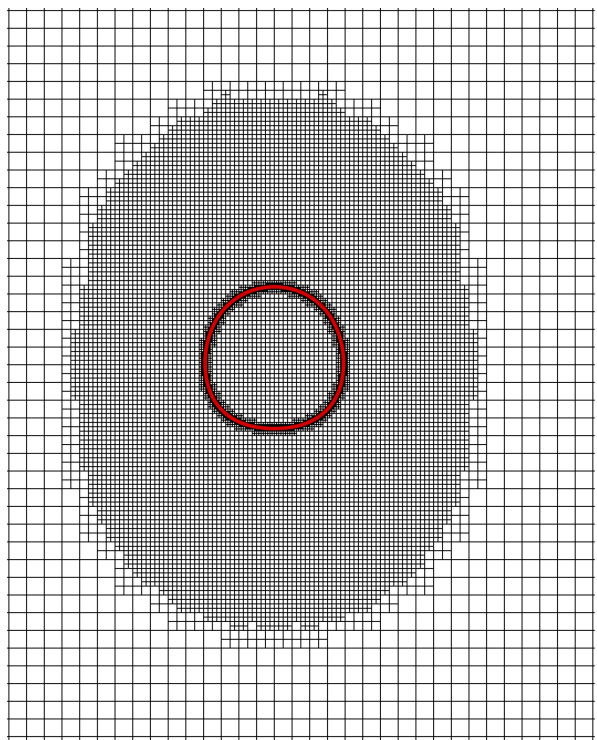

Coarser grid

Figure 2: The shape of the bubble along with the mesh are shown for (a) finer and (b) coarser grids. Adaptive grid refinement has been used in the interfacial and yielded regions. The smallest mesh size in the finer and coarser grids are 0.015 and 0.0625 , respectively. Note that the finer grid has been used to generate the results presented in the subsequent figures. The parameter values are $R e=70.71, B n=14.213, \mu_{r}=0.01, \rho_{r}=0.001, m=1$ and $B o=30$. 
(a)

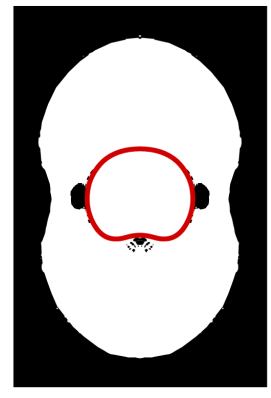

(b)

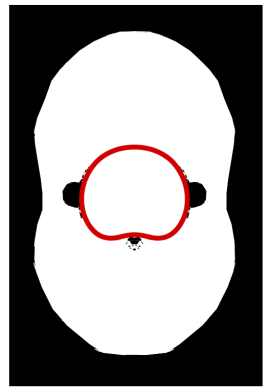

(c)

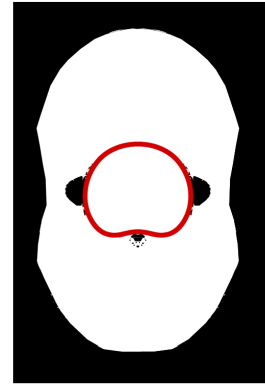

Figure 3: The unyielded region in the non-Newtonian fluid (shown in black) for different values of the regularized parameter, $\epsilon:$ (a) $\epsilon=0.01$, (b) $\epsilon=0.001$, (c) $\epsilon=0.0001$. The rest of the parameter values are $R e=70.71, B n=14.213, \mu_{r}=0.01, \rho_{r}=0.001, m=1$ and $B o=30$. 
(a)

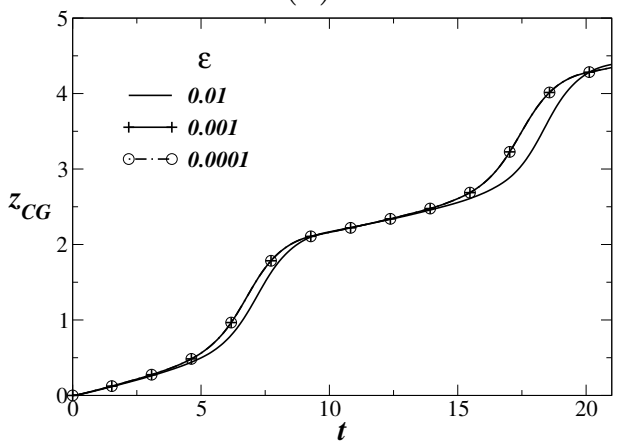

(b)

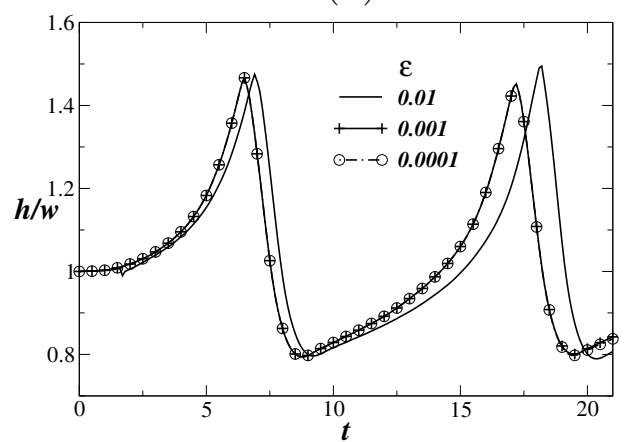

Figure 4: (a) Temporal variation of the center of gravity, (b) the aspect ratio of the bubble for different values of $\epsilon$. The rest of the parameter values are the same as those used to generate Fig. 3. 
(a)

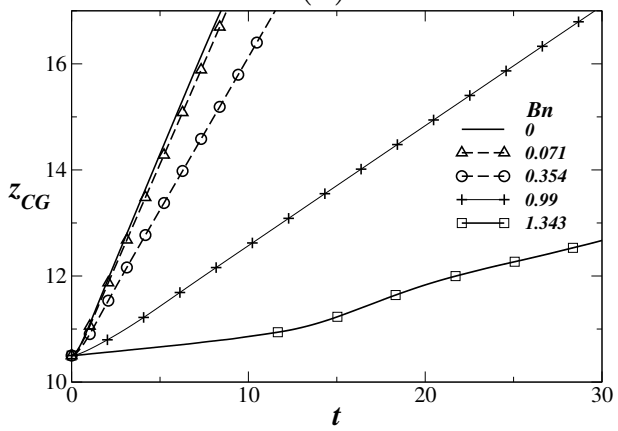

(b)

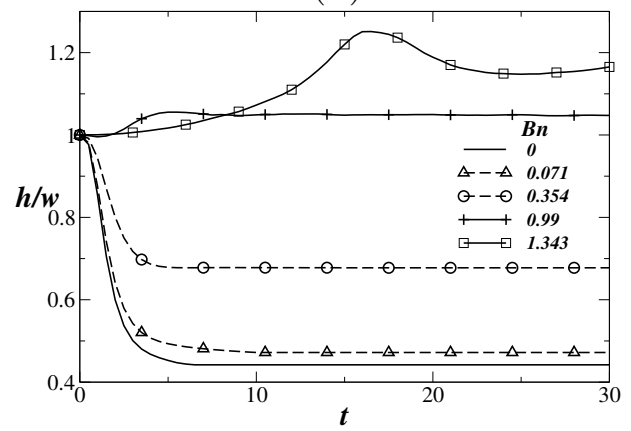

Figure 5: (a) Temporal variation of the center of gravity, (b) the aspect ratio of the bubble for different values of $B n$. The parameter values are $R e=7.071, \mu_{r}=0.01, \rho_{r}=0.001$, $m=1$ and $B o=10$. 
(a)

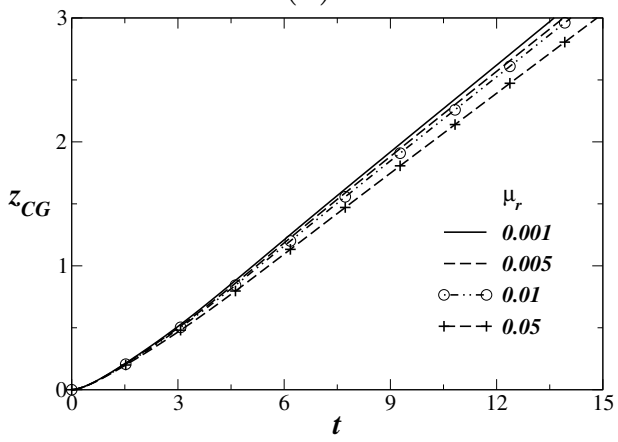

(b)

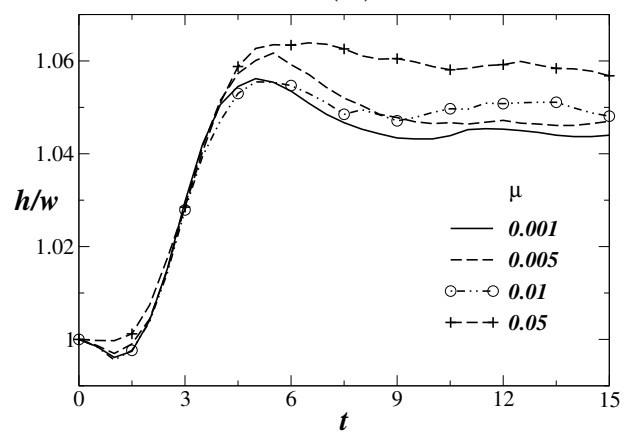

Figure 6: (a) Temporal variation of the center of gravity, (b) the aspect ratio of the bubble for different values of $\mu_{r}$. The parameter values are $R e=7.071, B n=0.99, \rho_{r}=0.001$, $m=1$ and $B o=10$. 
$B n=0$

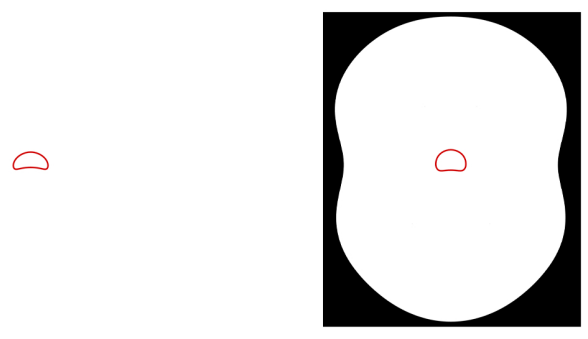

6

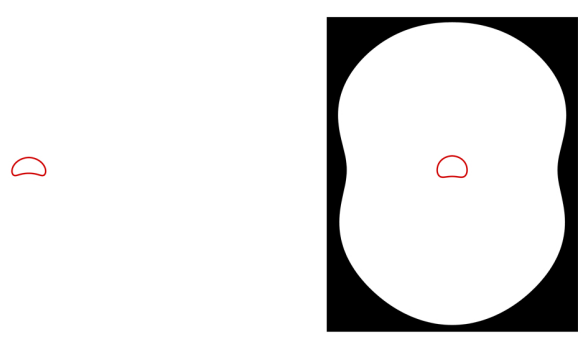

4

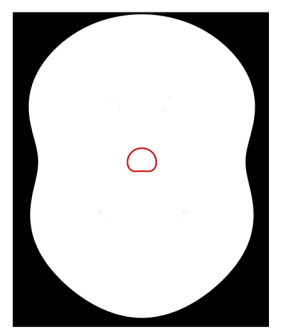

2

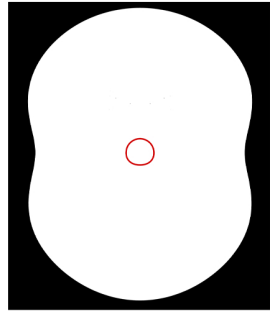

0.99

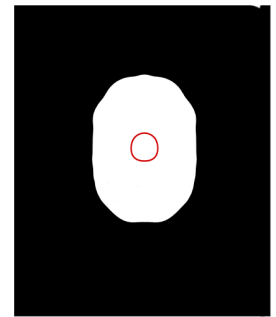

20

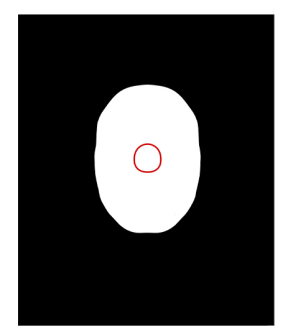

12
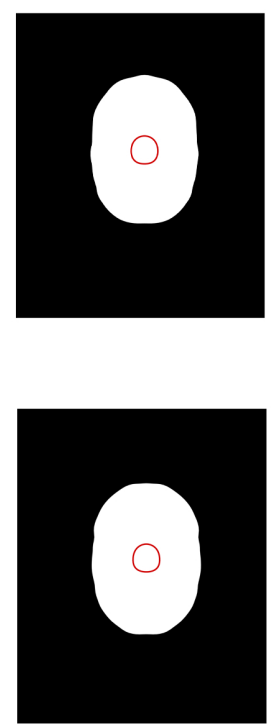
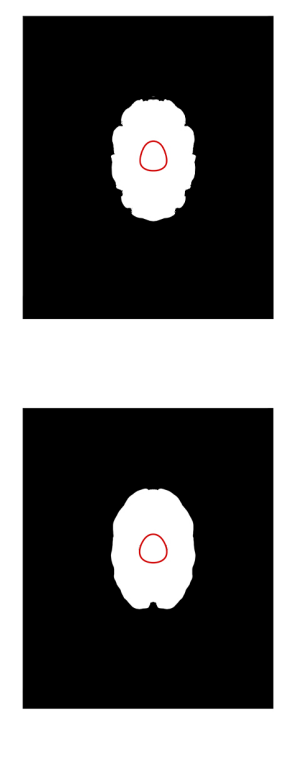

1.34

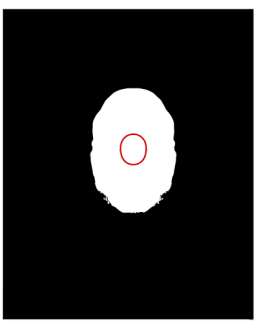

0

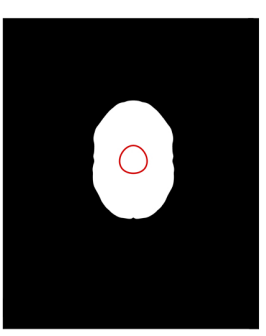

$$
t=1
$$

$$
t=4
$$

Figure 7: The evolution of the shape of the bubble (shown by red lines) and the unyielded region in the non-Newtonian fluid (shown in black) for different values of Bingham number. The results of the Newtonian case are shown for the comparison purpose. The rest of the parameter values are the same as those used to generate Fig. 5. 
(a)

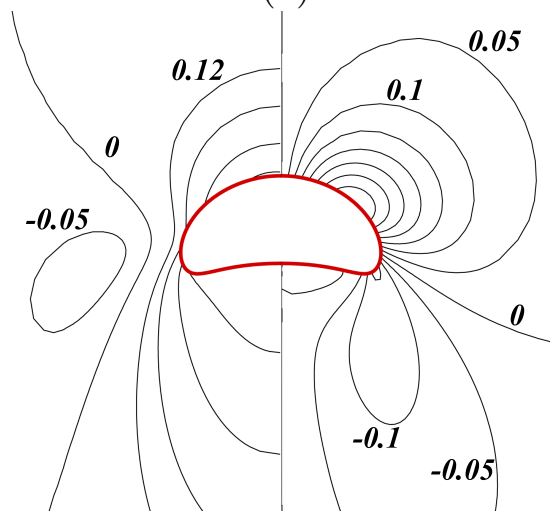

(c)

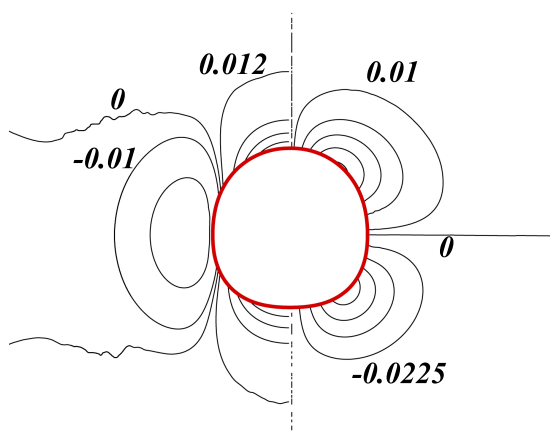

(b)

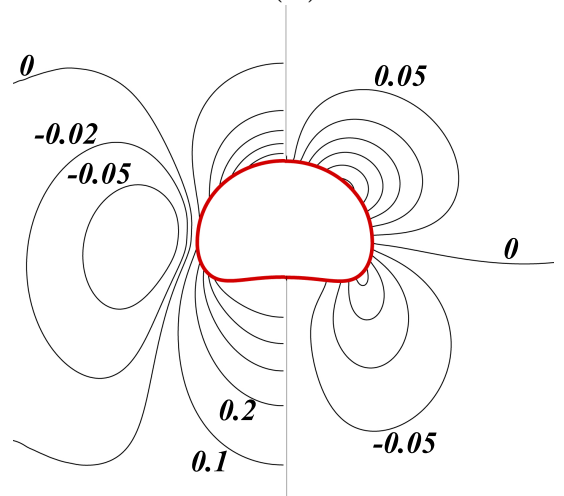

(d)

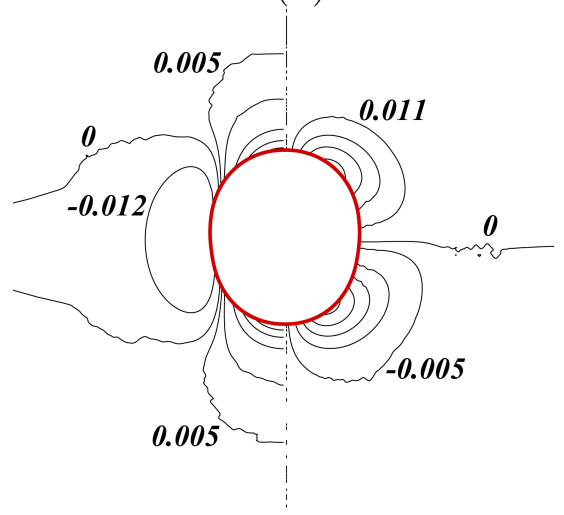

Figure 8: Contour plots for the radial (right) and axial (left) velocity components for (a) $B n=0$ at $t=6$ (Newtonian case), (b) $B n=0.354$ at $t=6$, (c) $B n=0.99$ at $t=20$ and (d) $B n=1.34$ at $t=20$. In each panel the shape of the bubble is shown by red line. The rest of the parameter values are the same as those used to generate Fig. 5 . 
(a)

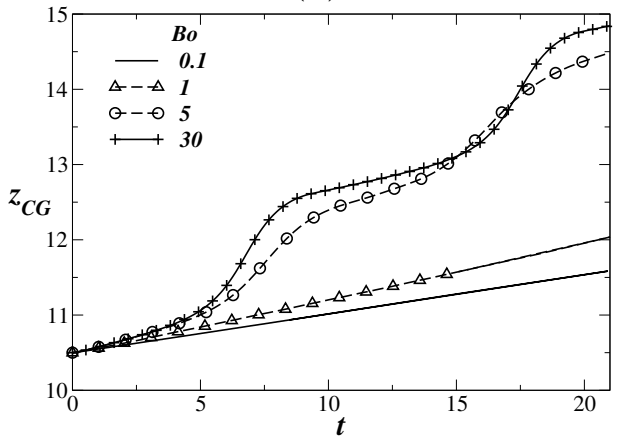

(b)

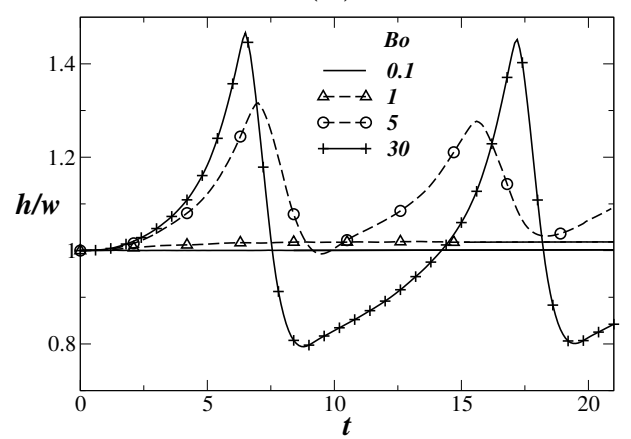

Figure 9: (a) Temporal variation of the center of gravity, (b) the aspect ratio of the bubble for different values of $B o$. The rest of the parameter values are $R e=70.71, B n=14.213$, $\mu_{r}=0.01, \rho_{r}=0.001$, and $m=1$. 


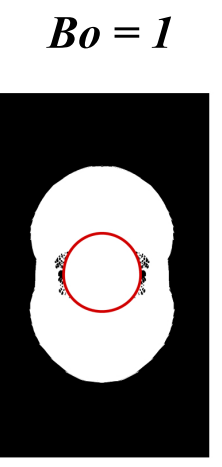

$$
B o=30
$$

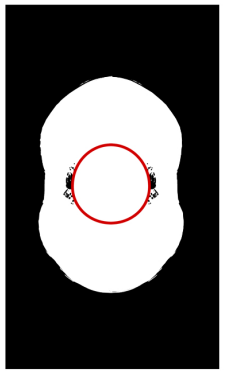

10
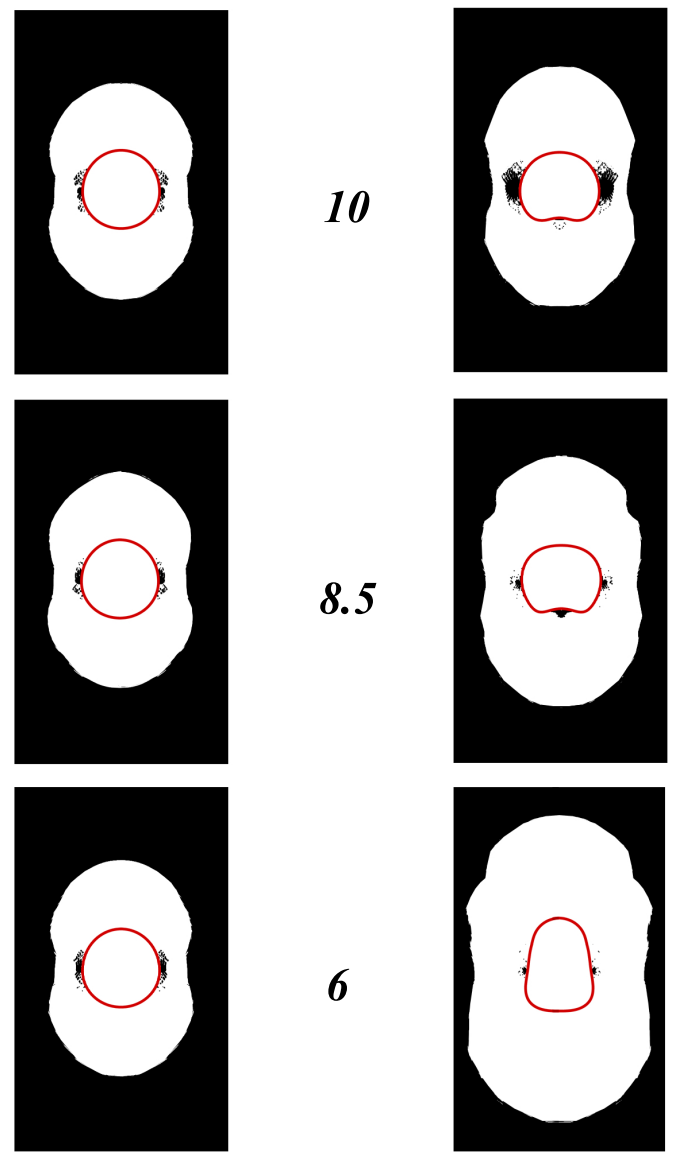

8.5

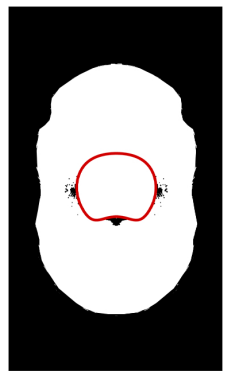

6
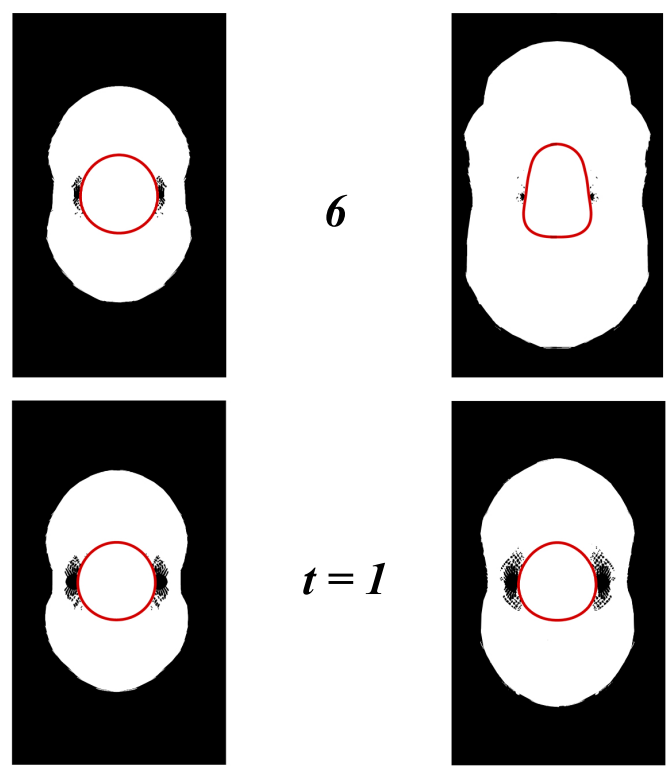

$$
t=1
$$

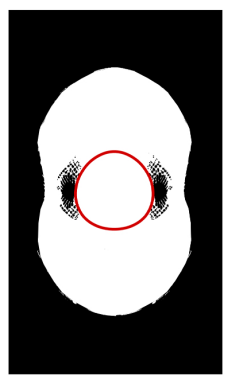

Figure 10: The evolution of the shape of the bubble (shown by red lines) and the unyielded regions in the Bingham fluid (shown in black) for different values of Bo. The rest of the parameter values are the same as those used to generate Fig. 9. 
(a)

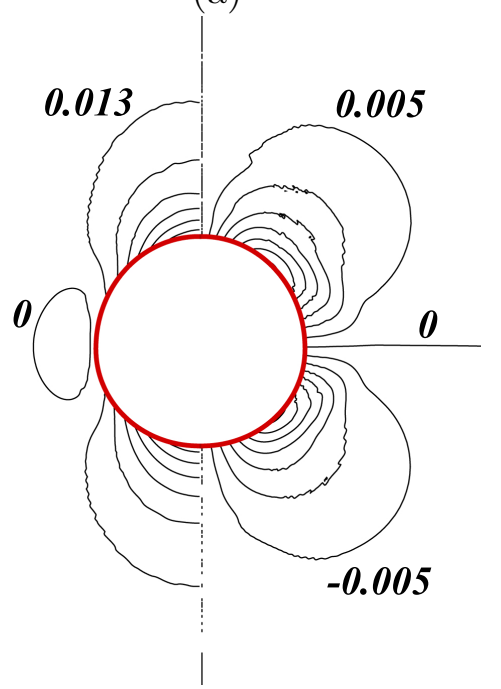

(c)

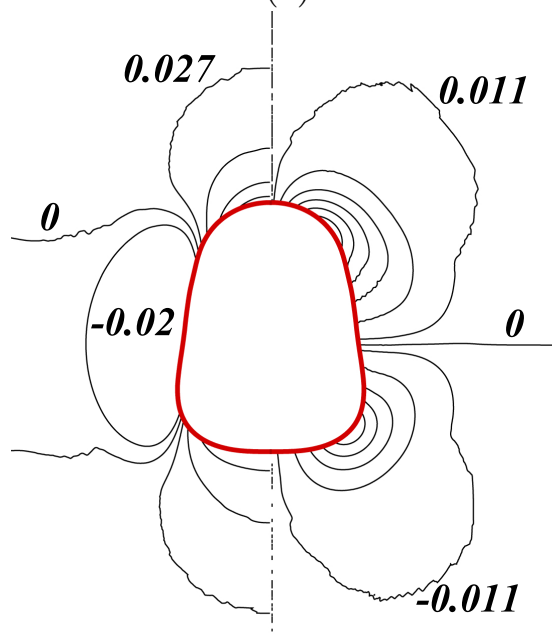

(b)

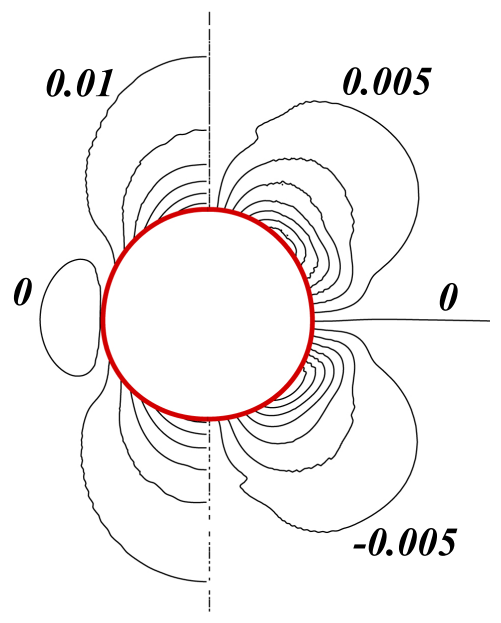

(d)

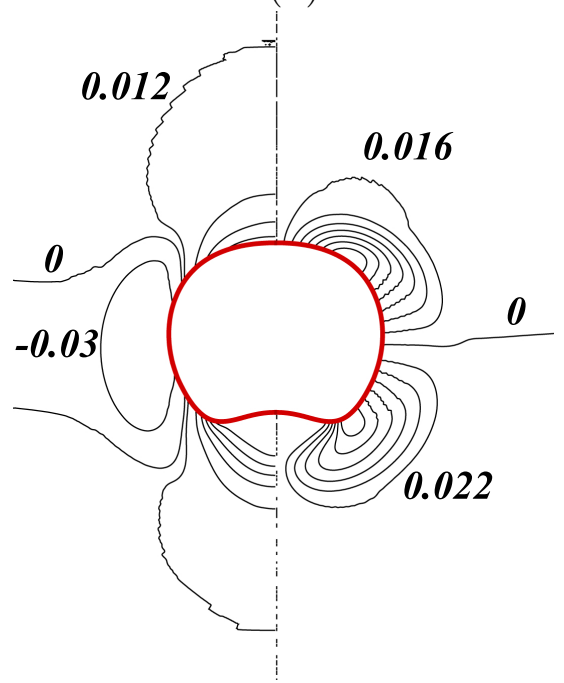

Figure 11: Contour plots for the radial (right) and axial (left) velocity components for (a) $B o=1$ at $t=6$, (b) $B o=1$ at $t=8.5$, (c) $B o=30$ at $t=6$ and (d) $B o=30$ at $t=8.5$. In each panel the shape of the bubble is shown by red line. The rest of the parameter values are the same as those used to generate Fig. 9. 